\title{
Erratum: Constraints on dark matter-nucleon effective couplings in the presence of kinematically distinct halo substructures using the DEAP-3600 detector [Phys. Rev. D 102, 082001 (2020)]
}

P. Adhikari, R. Ajaj, D. J. Auty, C. E. Bina, W. Bonivento, M. G. Boulay, M. Cadeddu, B. Cai, M. Cárdenas-Montes, S. Cavuoti, Y. Chen, B. T. Cleveland, J. M. Corning, S. Daugherty, P. DelGobbo, P. Di Stefano, L. Doria, M. Dunford, A. Erlandson, S. S. Farahani, N. Fatemighomi, G. Fiorillo, D. Gallacher, E. A. Garcés, P. García Abia, S. Garg, P. Giampa, D. Goeldi, P. Gorel, K. Graham, A. Grobov, A. L. Hallin, M. Hamstra, T. Hugues, A. Ilyasov, A. Joy, B. Jigmeddorj, C. J. Jillings, O. Kamaev, G. Kaur, A. Kemp, I. Kochanek, M. Kuźniak, M. Lai, S. Langrock, B. Lehnert, N. Levashko, X. Li, O. Litvinov, J. Lock, G. Longo, I. Machulin, A. B. McDonald, T. McElroy, J. B. McLaughlin, C. Mielnichuk, J. Monroe, G. Oliviéro, S. Pal, S. J. M. Peeters, V. Pesudo, M.-C. Piro, T. R. Pollmann, E. T. Rand, C. Rethmeier, F. Retière, I. Rodríguez-García, L. Roszkowski, E. Sanchez García, T. Sánchez-Pastor, R. Santorelli, D. Sinclair, P. Skensved, B. Smith, N. J. T. Smith, T. Sonley, R. Stainforth, M. Stringer, B. Sur, E. Vázquez-Jáuregui, S. Viel, A. C. Vincent, J. Walding, M. Waqar, M. Ward, S. Westerdale๑, J. Willis, and A. Zuñiga-Reyes

(DEAP Collaboration)

(Received 24 December 2021; published 19 January 2022)

DOI: 10.1103/PhysRevD.105.029901

In the article, the Non-Relativistic Effective Field Theory (NREFT) rate calculations were determined using the WIMpy_NREFT software [1], which was updated on September 29, 2021, to include a previously missing $\left(q / m_{N}\right)^{2}$ factor in the implementation. This update affects the results related to the $\mathcal{O}_{3}$ operator that now scales as $\left(q / m_{N}\right)^{4}$ instead of $\left(q / m_{N}\right)^{2}$. The corrections to Figs. 2, 6, 9, 10, and 11 are presented below. The couplings to $\mathcal{O}_{3}$ constrained by this analysis are higher than those reported in the article. Additionally:

(i) In Sec. VA, operator $\mathcal{O}_{3}$ is suppressed at low recoil energies, exhibiting now a peak around $50 \mathrm{keV}$ (Fig. 2).

(ii) The third paragraph in Sec. V B should read as follows: "The operator $\mathcal{O}_{3}$ is proportional to $\left(\mathrm{q} / \mathrm{m}_{N}\right)^{4}$, while $\mathcal{O}_{11}$ goes as $\left(q / m_{N}\right)^{2} \cdot \mathcal{O}_{3}$ is described by the $\Phi^{\prime \prime}$ multipole operator [discussed in Eqs. (9) and (10)], while $\mathcal{O}_{11}$ is described by $M$. Since the former operator is related to spin-orbit coupling, it couples to the two unpaired neutrons and proton holes in ${ }^{40} \mathrm{Ar}$, rather than to all 40 nucleons. This leads to a suppression of $\sim 10^{2}$ in addition to the extra $q^{2}$ suppression."

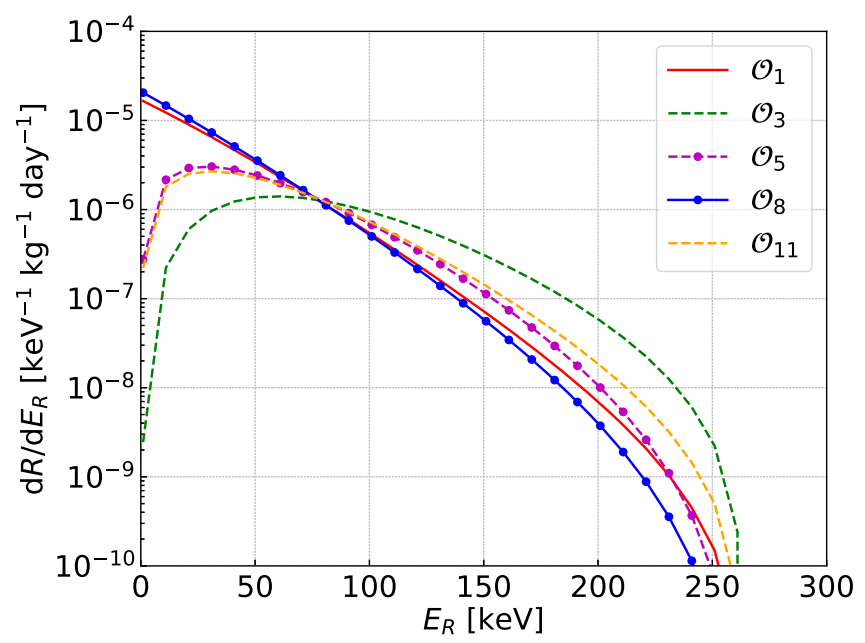

FIG. 2. Corrected recoil spectra for $\mathcal{O}_{3}\left(3.1 \times 10^{-38} \mathrm{~cm}^{2}\right.$, green). 


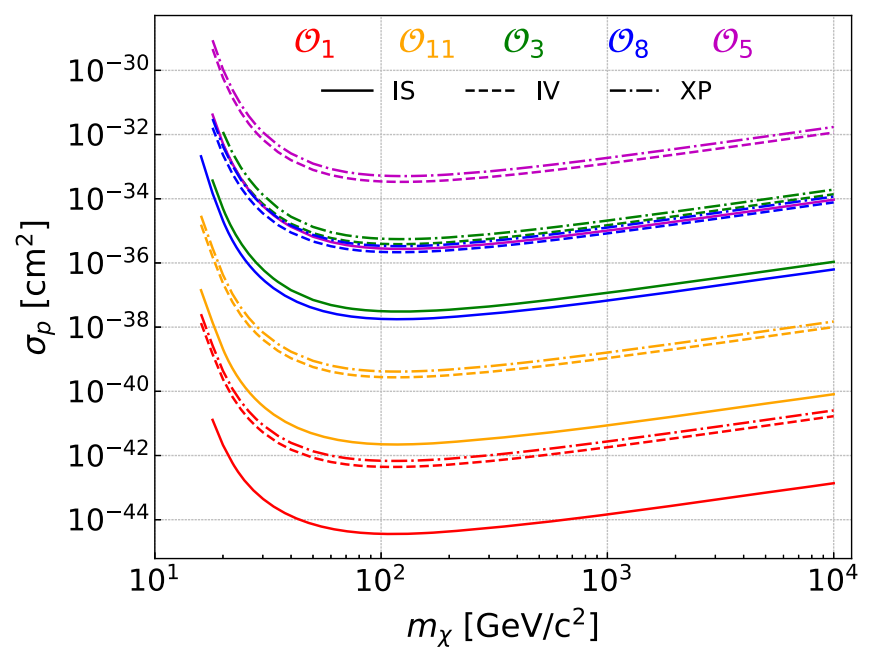

FIG. 6. Corrected upper limits (IS, IV, XP) for operator $\mathcal{O}_{3}$ (green).

(iii) In Sec. V F, the statement "Operators that introduce a factor of $q^{2}$ to the DM response function, such as $\mathcal{O}_{3}, \mathcal{O}_{5}$, and $\mathcal{O}_{11}$ change the shape of the recoil energy spectrum, compared to $\mathcal{O}_{1}$ " should read "Operators that introduce a factor of $q^{2}$ or $q^{4}$ to the DM response function, such as $\mathcal{O}_{3}, \mathcal{O}_{5}$, and $\mathcal{O}_{11}$ change the shape of the recoil energy spectrum, compared to $\mathcal{O}_{1}$."

(iv) The sentence in Sec. VI "Constraints on operators proportional to $v_{\perp}$ are weaker than those proportional to $q$, which are weaker than those proportional to neither" should read "Constraints on operators proportional to $v_{\perp}^{n}$ are weaker than those proportional to $q$ raised to the same power, which in turn are weaker than constant couplings."

(v) In Sec. VI, exclusion curves on $\mathcal{O}_{3}$ and data to reproduce its recoil energy spectra were uploaded to a new Zenodo version [2].

The updated rate calculations for all NREFT operators are also found to be in agreement with results from the dmdd (dark matter direct detection) software developed by Gluscevic et al. [3,4]. We thank Chris Cappiello for identifying and notifying us of the error in WIMpy_NREFT. 


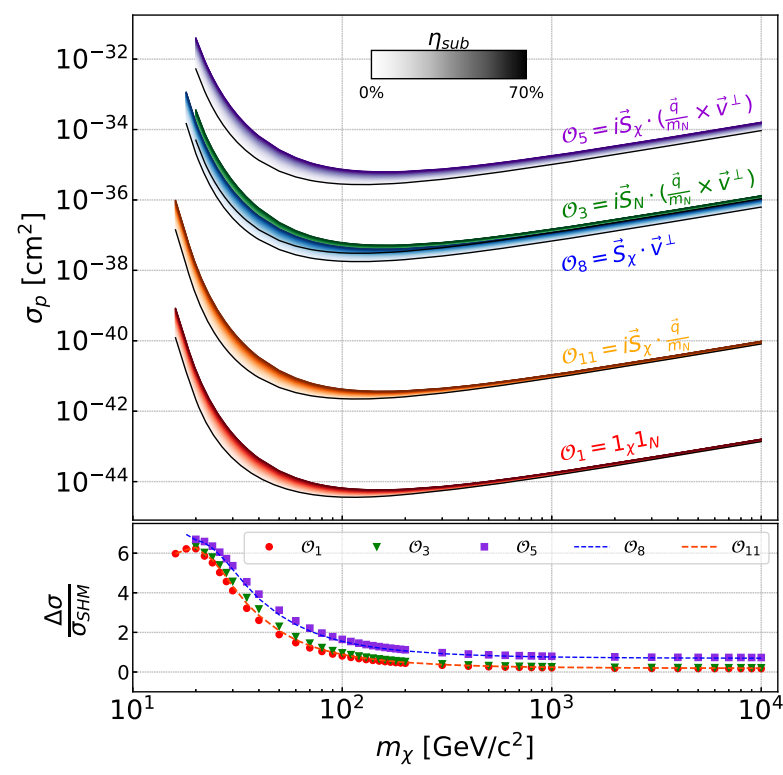

(a) Gaia Sausage (Necib)

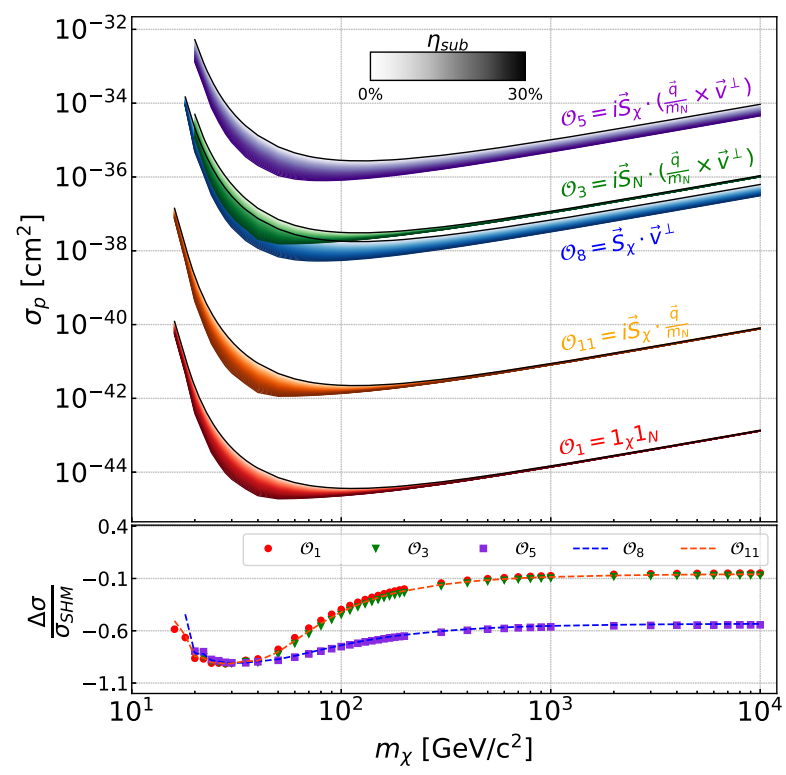

(c) G1 streams

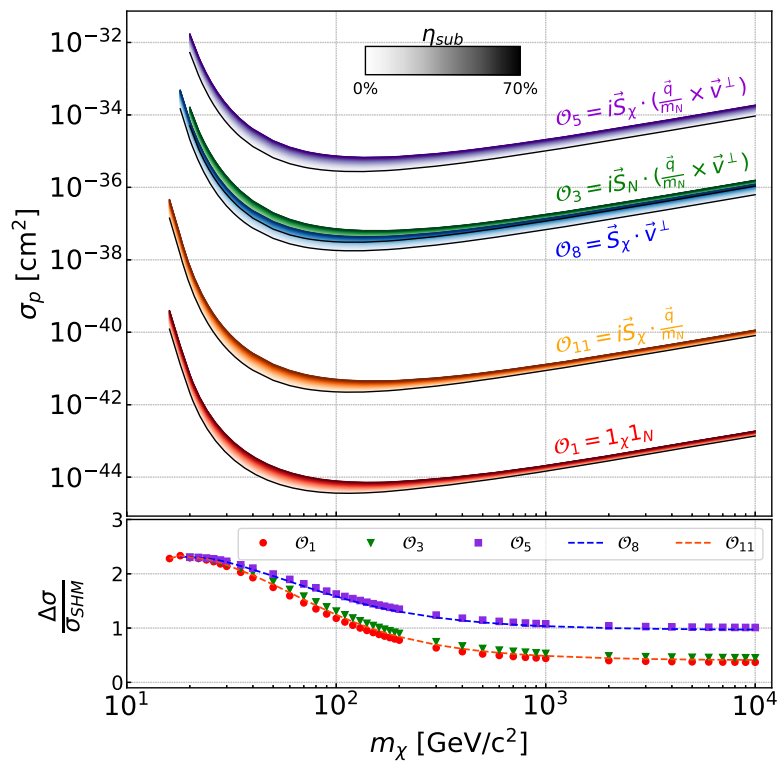

(b) Gaia Sausage (O'Hare)

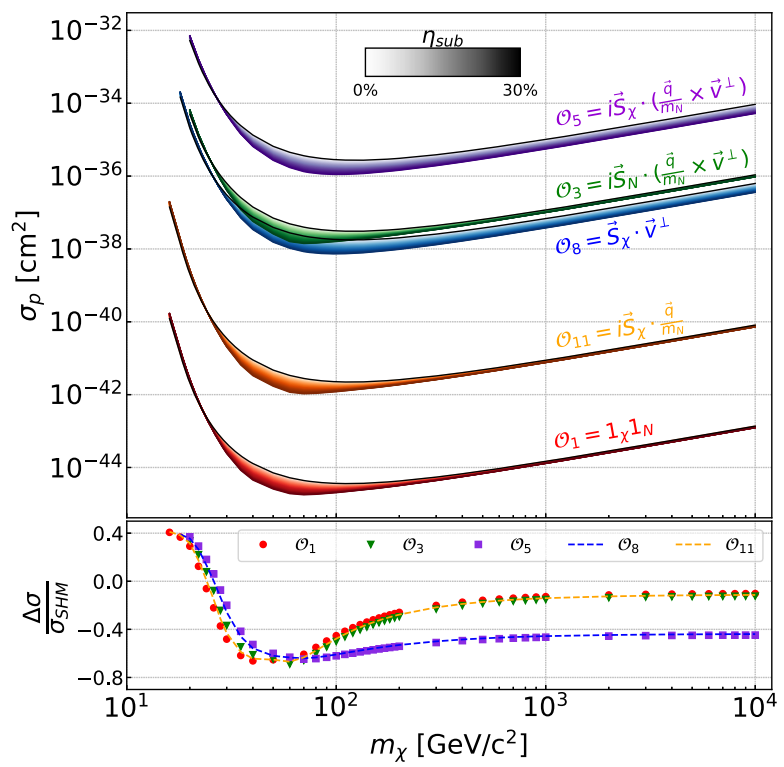

(d) G2 streams

FIG. 9. Corrected upper limits (90\% C.L.) for the $\mathcal{O}_{3}$ operator (green). 


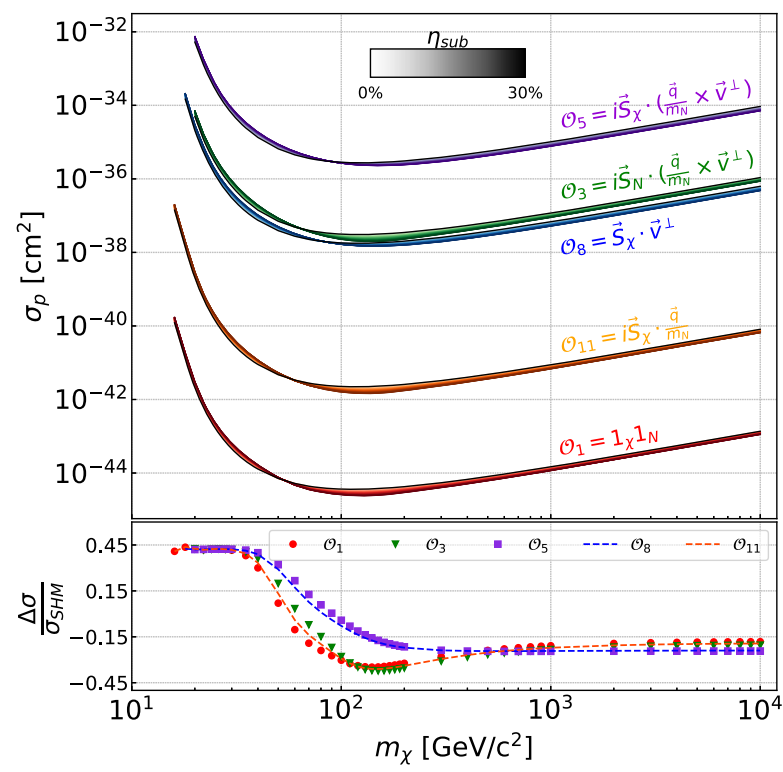

(a) G3 streams

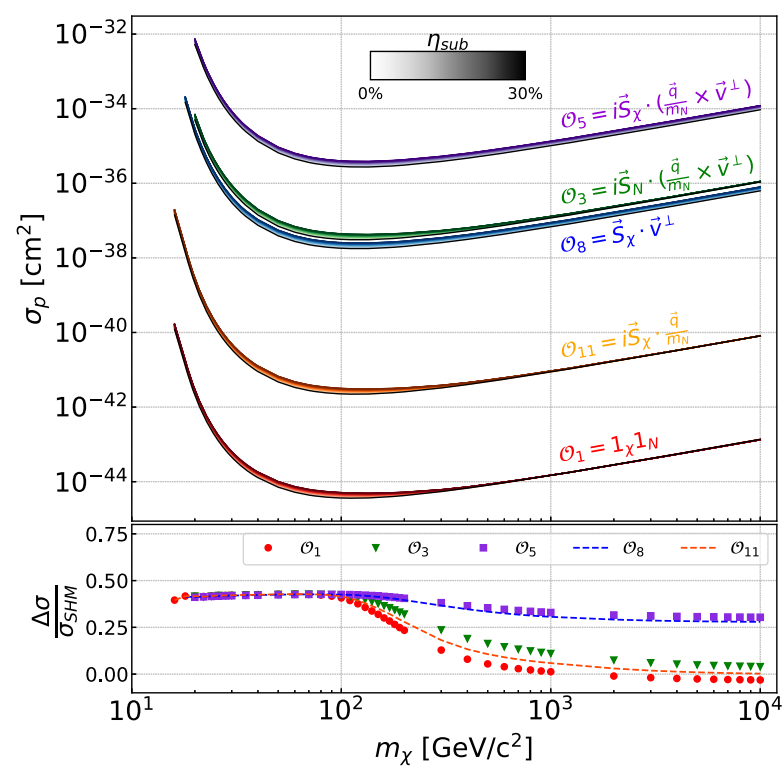

(c) G5 streams

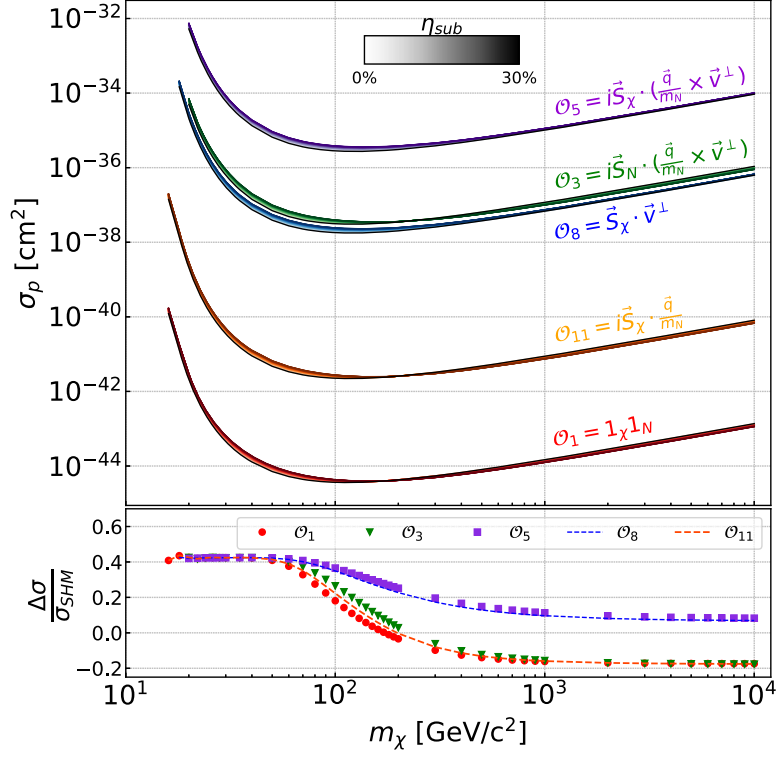

(b) G4 streams

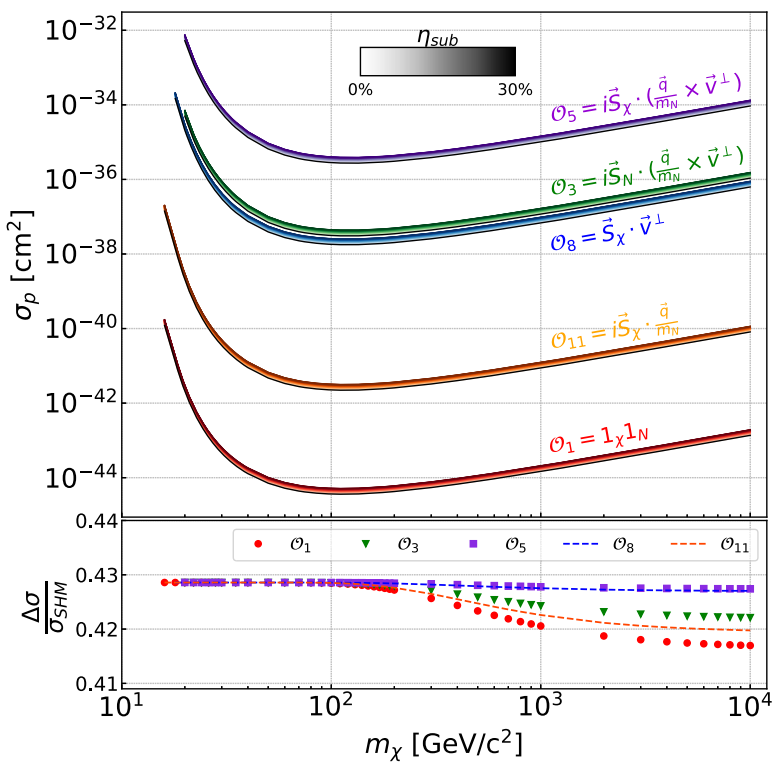

(d) G6 streams

FIG. 10. Corrected upper limits (90\% C.L.) for the $\mathcal{O}_{3}$ operator (green). 


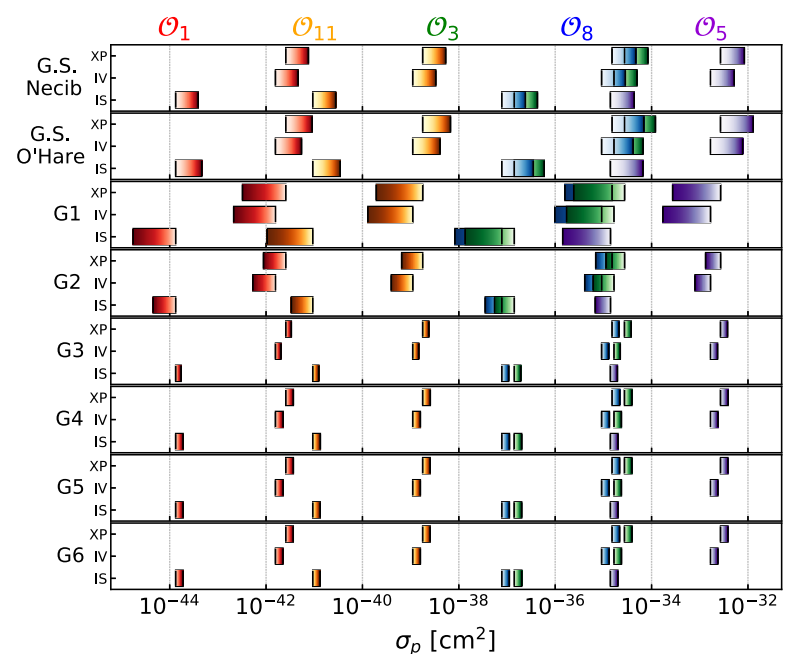

(a) $m_{\chi}=40 \mathrm{GeV} / c^{2}$

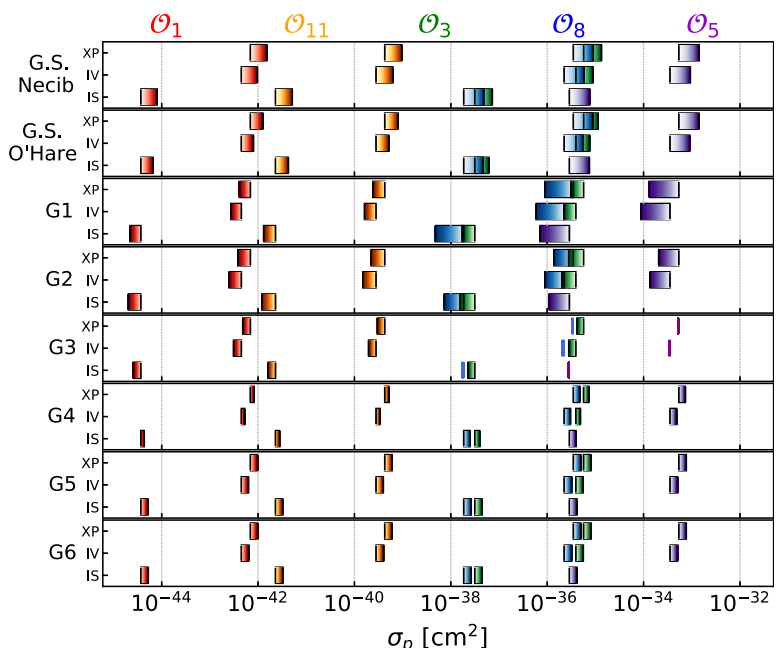

(b) $m_{\chi}=100 \mathrm{GeV} / c^{2}$

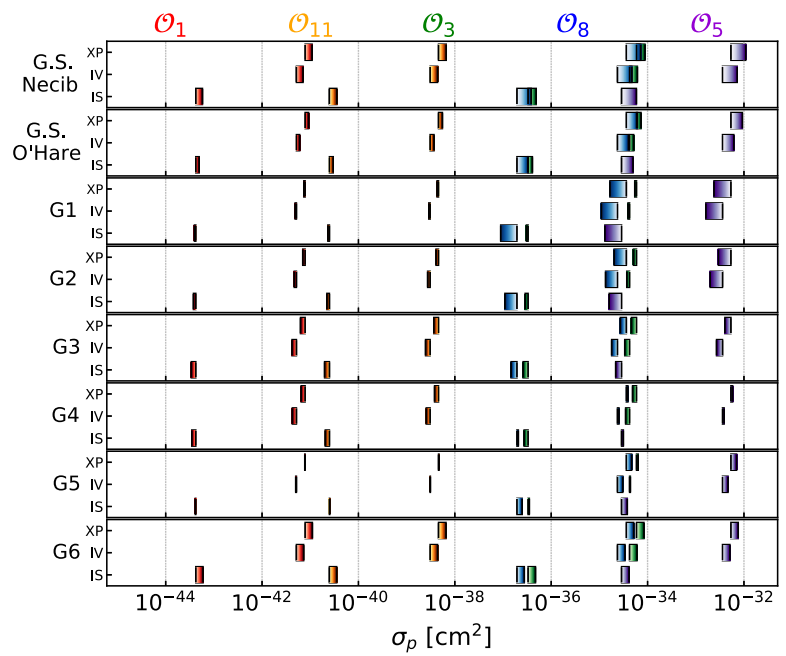

(c) $m_{\chi}=3 \mathrm{TeV} / c^{2}$

FIG. 11. Summary plots with corrected $\mathcal{O}_{3}$ constraints (90\% C.L.).

[1] B. J. Kavanagh and T. D. P. Edwards, computer code WIMPY_NREFT1.1, 2018, 10.5281/zenodo.1230503.

[2] DEAP-3600 Collaboration, Erratum: Constraints on dark matter-nucleon effective couplings in the presence of kinematically distinct halo substructures using the DEAP-3600 detector [Phys. Rev. D 102, 082001 (2020)], 10.5281/zenodo.5764847.

[3] V. Gluscevic, M. I. Gresham, S. D. McDermott, A. H. G. Peter, and K. M. Zurek, Identifying the theory of dark matter with direct detection, J. Cosmol. Astropart. Phys. 12 (2015) 057.

[4] V. Gluscevic and S. D. McDermott, dmdd: Dark matter direct detection, Astrophysics Source Code Library, ascl:1506.002, 2015, https://ui.adsabs.harvard.edu/abs/2015ascl.soft06002G. 